17 Dhadial, R K, Machin, A M, and Tait, S M, Lancet, 1970, 2, 20.

18 Underwood, P B, et al, Obstetrics and Gynecology, 1967, 29, 1.

19 Longo, L D, Science, 1976, 194, 523.

20 Suciu-Foca, N, et al, Lancet, 1974, 1, 1062.

21 Whitehead, R H, et al, Lancet, 1974, 1, 1232.

22 Silverman, N A, et al, Clinical and Experimental Immunology, 1975, 22, 285.

${ }^{23}$ Finklea, J F, et al, American Review of Respiratory Diseases, 1971, 104, 368.

24 Nymand, G, Lancet, 1974, 2, 1379.

${ }^{25}$ Rocklin, R E, et al, New England fournal of Medicine, 1976, 295, 1209.

\section{Tuberculosis of the female genital tract}

Tuberculosis of the genital tract is almost invariably secondary to disease elsewhere-usually in the lung, less frequently in the renal tract, and occasionally in a bone or joint. There may be a long latent period between the initial infection and its discovery in the genital tract; often little or no evidence of the primary lesion remains, or it may be totally inactive. A known pre-existing pulmonary lesion will make the interpretation of pelvic symptoms (which may be quite imprecise) relatively easy, but even direct questioning may fail to elicit a history of tuberculosis and examination may fail to show any trace of an earlier lesion. Clinicians need to remember that tuberculosis is still the most important communicable disease in the world-nearly half of India's population has at one time been infected. ${ }^{1}$ The incidence of reported disease in Britain has declined recently ${ }^{2}$ but a reservoir remains, and global population movements ensure that there will be a steady seeding for the foreseeable future.

Earlier surveys showed that the peak ages for genital tuberculosis were in the $20 \mathrm{~s}$ and 30 s. Today the age range of affected individuals with pulmonary disease has two peaks ${ }^{4}$-the lower one representing an immigrant group and the higher one the indigenous population. A wide scatter of ages, with a maximum incidence in the fourth decade, has been reported from New Zealand $^{5}$ for genital disease, which is often a chronic infection with abscess formation. The younger members of this group were immigrants from the Pacific Islands.

The Fallopian tube is the most common site of tuberculous pelvic infection, with spread to the uterine endometrium in $60 \%$ of cases. Less frequently organisms may be identified in the ovary, cervix, vagina, and vulva, but in virtually all cases the Fallopian tubes are affected as well. Most patients have bilateral lesions. Infection has usually been through the blood stream, but direct spread from other abdominal organs and the peritoneum is possible.

Most patients with genital tuberculosis present with infertility, abdominopelvic pain, or menstrual irregularity. Infertility is by far the most common of the three, and, though figures vary, up to $5 \%$ of those attending infertility clinics anywhere in the world will be found to have active disease. One-fifth of these will be entirely normal on clinical examination, and awareness that the disease is still with us is thus particularly important.

Though it may result from advanced active pulmonary disease, amenorrhoea is rarely a presenting symptom of genital tuberculosis. Malkani and Ragani, ${ }^{3}$ however, reported an incidence of amenorrhoea as high as $53 \%$ in some parts of India, attributing it to end organ failure from endometrial caseation. Menorrhagia and metrorrhagia are about as common as in other types of pelvic inflammatory disease, and there is a small but constant incidence of postmenopausal bleeding.
Genital tuberculosis is best diagnosed by histological examination of uterine endometrium taken before menstruation, with confirmation from culture or animal inoculation. Repeated examination of menstrual blood, collected with either a vaginal speculum or a cervical cup, may be useful. Changes may be seen in the hysterosalpingogram ${ }^{6}$ but these are not specific, and the procedure has found little favour in $\stackrel{\varrho}{c}$ Britain. Laparoscopy is contraindicated if active pelvic disease is suspected, but some cases may be identified in this $\frac{\vec{\rho}}{+}$ way by chance.

Treatment is straightforward: the drug regimens used so $\frac{\bar{D}}{\bar{D}}$ successfully for pulmonary lesions are also applied to genital $\frac{\bar{\phi}}{\mathrm{D}}$ tuberculosis. The outcome of such treatment, however, is nowhere as well documented as it is in pulmonary disease.

${ }^{1}$ Schaefer, G, Clinical Obstetrics and Gynecology, 1970, 13, 965.

2 Department of Health and Social Security, On the State of the Public Health 1975. London, HMSO, 1976.

${ }^{3}$ Malkani, P K, and Rajani, C, Indian Fournal of Medical Sciences, 1954, 8, 684

4 Stead, W W, in Harrison's Principles of Internal Medicine, 6th edn, ed $M$ Wintrobe et al, p 865. New York, McGraw Hill, 1970.

${ }^{5}$ Hutchins, C J, British fournal of Obstetrics and Gynaecology, 1977, 84, 534

${ }^{6}$ Ekengren, K, and Rydén, A B V, Acta Radiologica, 1950, 34, 193.

\section{Breast lumps in adolescent girls}

Public awareness of breast cancer has been heightened recently, largely through popular journalism, and in consequence a steady trickle of frightened young girls are attending surgical outpatients and specialist breast clinics. Many of them have cyclical mastalgia, nodularity, or asymmetry, but a small proportion will indeed present with breast lumps. How should doctors manage these breast lumps in teenage girls, and are they ever any cause for concern?

Cancer of the breast under the age of 30 is extremely rare, accounting for only $1.2 \%$ of the 6000 cases in Haagensen's series, ${ }^{1}$ for example; and carcinoma under the age of 20 might be considered a medical curiosity, with only seven cases among the series of over 70000 patients dying from the disease reported by Pirquets. ${ }^{1}$ Stone et al, writing from a clinic in New York, recently described 143 adolescent girls presenting with breast lumps over 14 years. ${ }^{2}$ As might be expected, over $70 \%$ proved to be fibroadenomas on biopsy. There was one case of infiltrating duct adenocarcinoma and two cases of cystosarcoma. The remainder were a variety of benign conditions including cysts and inflammatory lesions. On the basis of this experience the New York surgeons recommended careful observation and reassurance for two complete menstrual cycles with surgical excision if the mass persists.

At first sight this seems reasonable advice, given that cancer of the breast under the age of 20 is so rare and that when they do arise such cancers are said to have a comparatively benign course. ${ }^{1}$ Nevertheless, a recent report in the Lancet $^{3}$ described a 15-year-old schoolgirl who presented with a breast lump; it was diagnosed as a fibroadenoma but turned out to be an invasive carcinoma with extensive spread to the axillary nodes. She later died with massive hepatic metastases.

The safer policy, then, is for the surgeon to carry out an immediate biopsy on all breast lumps that appear in the breasts of postpubertal girls, if only to give them and their parents 